\title{
Pneumoperitoneum resulting from pneumatosis cystoides intestinalis: a rare complication of massive colonic dilatation
}

\author{
Kian-Peng J. Ong $\cdot$ Kheng-Hong Ng • \\ Kiat-Hon Lim $\cdot$ Su-Chong A. Low $\cdot$ \\ Kong-Weng Eu
}

Received: 16 December 2009/Accepted: 21 December 2009/Published online: 11 February 2010

(C) Springer-Verlag 2010

\begin{abstract}
A 69-year-old man presented with intestinal obstruction and peritonism. Chest and abdominal X-rays showed massive pneumoperitoneum with large bowel obstruction (Figs. 1, 2). Exploratory laparotomy revealed closed loop obstruction secondary to sigmoid volvulus with extensive pneumatosis cystoids intestinalis of non-dilated small bowel (Figs. 3, 4),
\end{abstract}

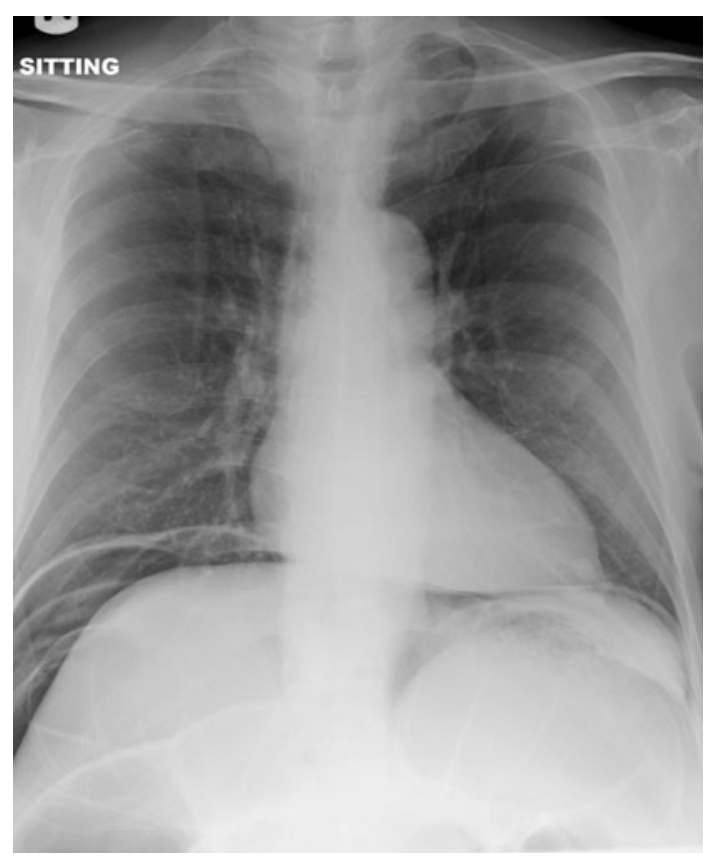

Fig. 1 Erect chest X-ray showing pneumoperitoneum

K.-P. J. Ong · K.-H. Ng · K.-H. Lim · S.-C. A. Low ·

K.-W. Eu (ه)

Singapore General Hospital, Singapore, Singapore

e-mail: eu.kong.weng@sgh.com.sg without evidence of perforation. We postulate that rupture of a submucosal cyst in the small bowel resulted in pneumoperitoneum. Total colectomy was performed, with ileocecal anastomosis and defunctioning ileostomy. Histology confirmed pneumatosis cystoides intestinalis of small bowel without full-thickness perforation (Figs. 5, 6).

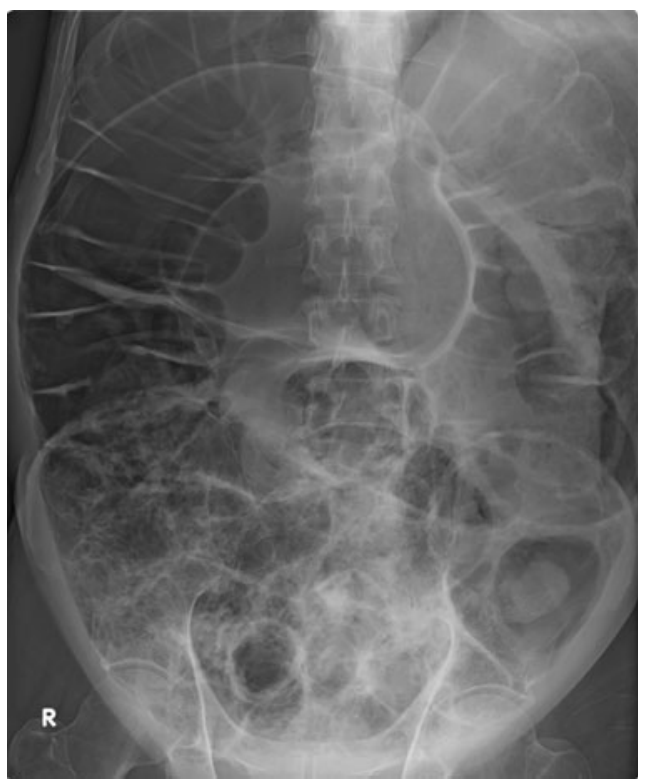

Fig. 2 Supine abdominal X-ray showing grossly dilated large bowel and multiple, clustered, small gaseous lucencies in the right lower abdomen, probably caused by air within the bowel wall, characteristic of pneumatosis intestinalis 


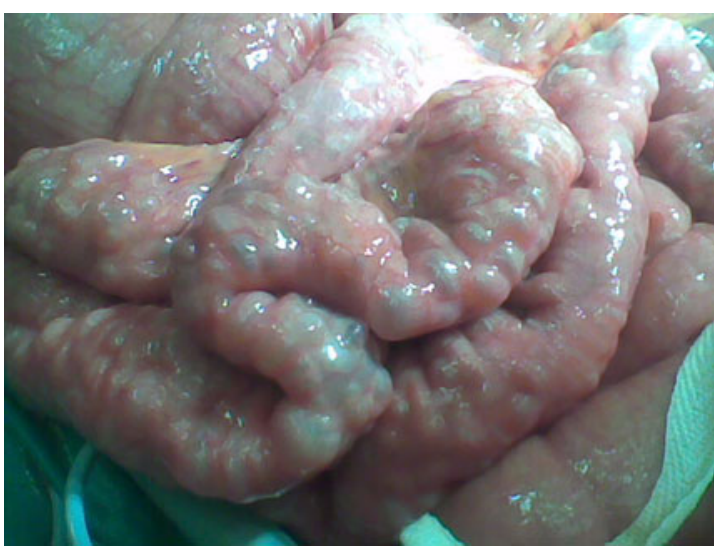

Fig. 3 Intraoperative image showing extensive pneumatosis intestinalis of the non-dilated distal small bowel

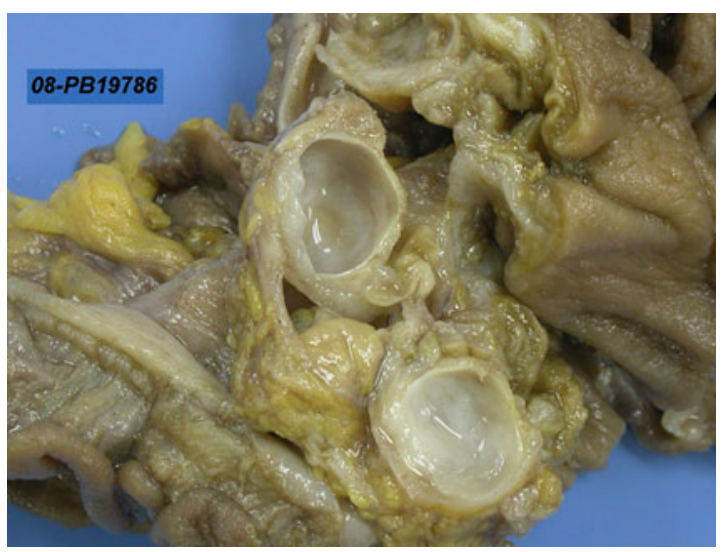

Fig. 4 Gross image showing cystic air bubbles in the submucosa

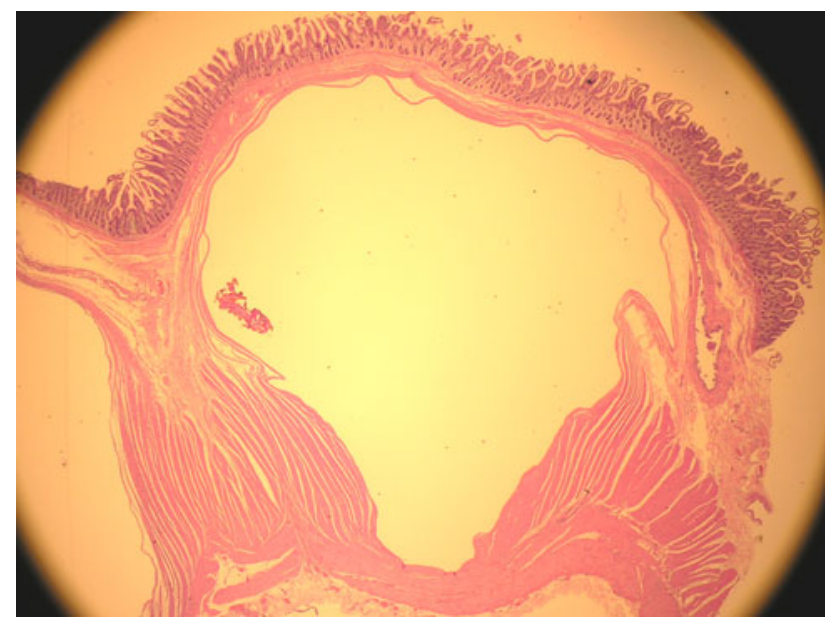

Fig. 5 Low-power microscopic view showing large cystic lesion in the submucosa

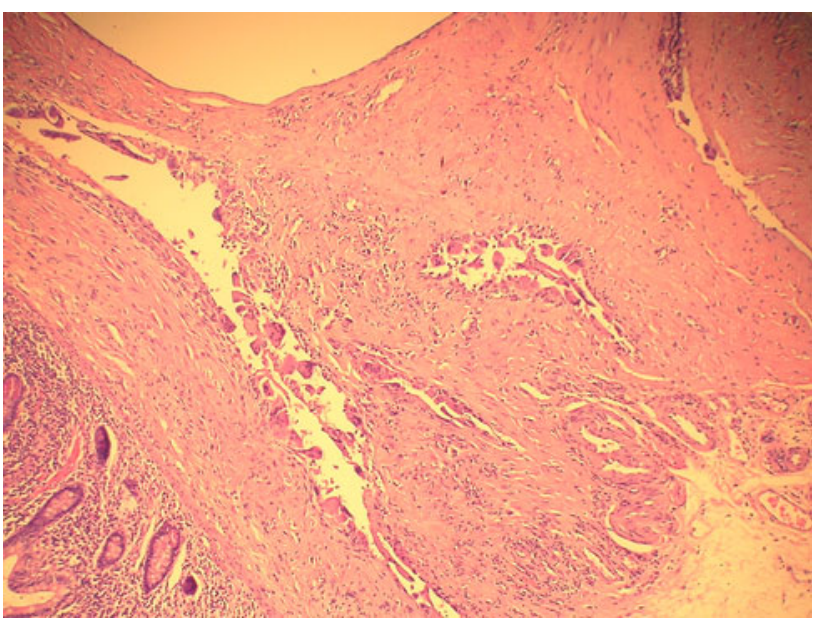

Fig. 6 High-power microscopic view showing foreign body giant cells lining the cystic spaces 